\title{
Ciclo de Mejora en el Aula aplicado en la Asignatura "Derecho de Empresa" del Primer Curso del Grado de Relaciones Laborales y Recursos Humanos
}

\author{
Improvement Cycles in \\ Classroom-ICIC) applied in the \\ subject "Business Law" of the \\ First Year of the Degree in Labor \\ Relations and Human Resources
}

Ma deL CARMEN VIDA CARRIÓN

ORCID: https://orcid.org/0000-0001-6417-7786

Universidad de Sevilla.

Facultad de Derecho.

Departamento de Derecho Civil

e Internacional Privado.

mvida@us.es

Fecha de recepción del artículo: 10/7/2019

Fecha de aceptación del artículo: 18/7/2019

DOI: http://dx.doi.org/10.12795/9788447221912.052

Pр.: $1203-1223$ 


\section{Resumen:}

En el contexto y desarrollo del Programa de Formación e Innovación del Profesorado de la Universidad de Sevilla realizado en el Curso 2018/2019 se ha procedido a la implementación en la Asignatura de Derecho de Empresa, para los Alumnos de primer Curso del Grado de Relaciones Laborales y Recursos Humanos, de la metodología de Aprendizaje Basado en Proyectos (ABP) en base a los principios que los apoyan y que se expondrán en el presente Artículo, llevándolo al campo práctico con la aplicación de un Ciclo de Mejora en el Aula donde se ha impartido la Asignatura referida. La ejecución del Ciclo de Mejora se ha desarrollado con las metodologías de clase, el tratamiento previo de los contenidos y la evaluación y calificación de los resultados finalmente obtenidos en base a parámetros de evaluación teórico-prácticos abordando los conocimientos y modelos mentales de los estudiantes, sus capacidades y aptitudes, que en este grupo en particular han sido relevantes en cuanto a la comparación progresiva de los resultados obtenidos en relación a las anteriores evaluaciones, por cuanto en un porcentaje alto, los Alumnos que han participado en la presente investigación de mejora, se encontraban en convocatorias muy avanzadas, casual peculiaridad que ha posibilitado excepcionalmente la comparación estructural en el cambio de aprendizaje y la valoración más que positiva del resultado obtenido y su análisis.

Palabras clave: Derecho de Empresa, Grado en Dirección y Administración de Empresas, Grado de Relaciones Laborales y Recursos Humanos, Docencia Universitaria, Experimentación Docente Universitaria, Aprendizaje Basado en Proyectos.

Jornadas de Formación e Innovación Docente del Profesorado | № 2 (2019) Esta obra se distribuye con la licencia Creative Commons Reconocimiento-NoComercial-SinObraDerivada Internacional (CC BY-NC-ND 4.0.) 
Abstract:

In the context and development of the Teacher Training and Innovation Program of the University of Seville conducted in the 2018/2019 Course has been implemented in the Business Law Subject, for First Year Students of the Degree in Labor Relations and Human Resources, the methodology of Project-Based Learning (PBL) based on the principles that support them and that will be presented in this Article, taking it to the practical field with the application of a Cycle of Improvement in the Classroom where imparted the Subject referred. The execution of the Improvement Cycle has been developed with the class methodologies, the previous treatment of the contents and the evaluation and qualification of the results finally obtained based on theoretical-practical evaluation parameters addressing the knowledge and mental models of the students, their abilities and aptitudes, which in this particular group have been relevant in terms of the progressive comparison of the results obtained in relation to the previous evaluations, as in a high percentage, the students who have participated in the present research for improvement, they were in very advanced calls, a casual peculiarity that has exceptionally enabled the structural comparison in the change of learning and the more than positive evaluation of the result obtained and its analysis.

Key words: University Teaching, University Teaching Experimentation, Project Based Learning (PBL).

Jornadas de Formación e Innovación Docente del Profesorado | № 2 (2019) Esta obra se distribuye con la licencia Creative Commons 


\section{Breve descripción del contexto de la intervención}

Las clases experimentales se han aplicado en la Asignatura cuatrimestral de Derecho de Empresa, para los Alumnos de primer Curso del Grado de Relaciones Laborales y Recursos Humanos, mediante el desarrollo de un Ciclo de Mejora en el Aula, en el que han intervenido simultáneamente las tres variables fundamentales del proceso de enseñanza-aprendizaje, a saber, contenidos, metodología y evaluación, abarcando la asignatura completa a lo largo de todo el cuatrimestre analizando los resultados finalmente obtenidos en base a parámetros de evaluación teórico-prácticos. En este Ciclo de mejora se ha propiciado una circunstancia casual que ha posibilitado excepcionalmente la exploración de un análisis comparativo estructural cuanto menos privilegiado de los modelos mentales reales de los estudiantes, sus capacidades y aptitudes, habida cuenta que un porcentaje alto de los Alumnos que han participado en las clases experimentales se encontraban en convocatorias muy avanzadas ya agotadas de la asignatura en cuestión, por lo tanto habían sido Alumnos en cuanto a esta misma asignatura de distintos métodos didácticos y de evaluación que cada docente anterior había tenido por conveniente ejecutar y que ellos habían adaptado a distintos sistemas de estudio con un resultado de calificación desfavorable abocado a las últimas convocatorias agotadas, lo que conllevaba un modelo mental de aprendizaje y un comportamiento aptitudinal dificil de desectructurar de cara a la experimentación pretendida, pero que a la inversa ha ofrecido a este estudio un campo real de comparación en los avances obtenidos y la incidencia en los mismos del Ciclo de Mejora implantado en el Aula.

De los 42 Alumnos matriculados, han asistido regularmente a clase 28 Alumnos que han participando en el Ciclo de Mejora en el Aula, examinándose de ellos finalmente 27, aprobando todos a excepción de un Alumno, y de los cuales: 7 Alumnos eran de 1a Convocatoria, 7 Alumnos 
eran de 2a Convocatoria, 3 Alumnos eran de 3a Convocatoria, 3 Alumnos eran de 4a Convocatoria, 6 Alumnos eran de 5 a Convocatoria y 1 Alumno era de 6 a Convocatoria. Siendo que el resultado final, a nivel de calificación, arroja el siguiente resultado:

\section{Diseño previo del Ciclo de Mejora en el Aula}

\section{Mapa de contenidos y problemas que describen los aprendizajes deseables y los núcleos de trabajo en el Aula}

El contenido del temario en este Ciclo de mejora en el Aula ha sido "El Derecho de Empresa", inmerso en el Programa docente aprobado por el Departamento de Derecho Civil e Internacional Privado de la Facultad de Derecho de la Universidad de Sevilla para la Asignatura cuatrimestral de Derecho de Empresa a impartir en el Primer Curso del Grado de Relaciones Laborales y Recursos Humanos.

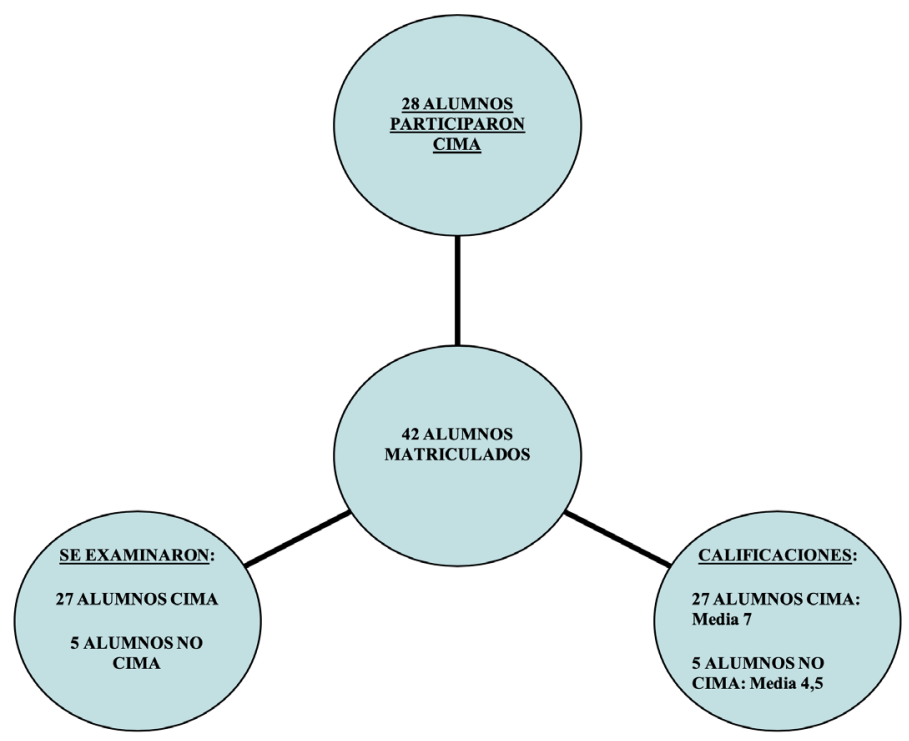

Figura 1: Proyección del resultado de calificaciones.

Jornadas de Formación e Innovación Docente del Profesorado | № 2 (2019) Esta obra se distribuye con la licencia Creative Commons Reconocimiento-NoComercial-SinObraDerivada 
El temario completo de la asignatura dentro del contexto del Programa se compone de 14 temas, a impartir en el segundo Cuatrimestre del Curso Académico 2018/2019, y que se podrían clasificar en Cuatro Bloques temáticos piramidales íntimamente relacionados entre y sí planteados en un orden de prelación consecuente con un aprendizaje estructural, por cuanto los primeros temas a desarrollar son el pilar de conocimiento necesario sobre los que construir el conocimiento de los temas sucesivos conforme a los dos siguientes parámetros.

I- Se han determinado los tipos de contenidos: Teóricos, relacionados con la metodología de investigación de la asignatura y asociados a principios y valores vinculados con problemas prácticos sobre el contenido de ella, y procedimentales, relacionados con la ejecución de estrategias técnicas y habilidades conducentes a la resolución de los problemas vinculados a las cuestiones que suscita la teoría; y por último contenidos actitudinales relacionados con las experiencias internas y subjetivas que implican valorar la teoría desde la perspectiva social y su aplicación real.

II- Se han relacionado contenidos y clasificado, estableciendo a qué tipo pertenece cada uno y razonando por qué clasificamos estos tipos de contenidos en la forma ejecutada y no desglosados en otros distintos, resultando finalmente los siguientes Cuatro Bloques temático teórico prácticos:

Contenidos teóricos y actitudinales:

Bloque 1: Derecho de la empresa y actividad empresarial. (Temas del 1 al 7).

Bloque 2: Empresa, empresario y sociedades mercantiles. (Temas del 8 al 11)

Contenidos procedimentales:

Bloque 3: Instrumentos jurídicos privados en el tráfico empresarial. (Temas del 12 al 13)

Bloque 4: Derecho Concursal. (Tema 14)

Jornadas de Formación e Innovación Docente del Profesorado I № 2 (2019) Esta obra se distribuye con la licencia Creative Commons 
De esta manera se han establecido contenidos teóricos y actitudinales desarrollados a través de contenidos procedimentales sobre como ejercer en la práctica los derechos subjetivos de la empresa, asociados a principios y valores vinculados con ellos, por cuanto los Derechos subjetivos son reconocidos legalmente para ejercerlos en la sociedad conforme al ordenamiento jurídico que a la vez está inspirado en los propios valores objetivos de Derecho Natural y de la sociedad.

La novedad de este ciclo de mejora ha consistido en sustituir el cuestionario inicial y final por una cuestión inicial y la posterior la elaboración de mapas de contenidos de la materia por parte de los estudiantes con su aplicación en un caso práctico real, la elaboración de su propia Empresa. A partir del análisis la cuestión inicial y de los mapas iniciales se ha construido una escalera inaugural con los diferentes modelos mentales de los estudiantes y sus porcentajes y se ha comparado con la escalera que se construya a partir del mapa final y el trabajo práctico real desarrollado. Todo ello ha permitido un seguimiento durante el proceso al analizar los mapas intermedios, teniendo como base de comparación el evaluador el ya estructurado mapa de contenido que se expondrá seguidamente, en base al ciclo de mejora en el Aula conceptuado en el mapa que se adjunta como Anexo I.

Teniendo por defecto esa base de clasificación de contenido, piensan clasificando, y se realiza inicialmente un mapa de contenido provisional consistente en la siguiente clasificación de la materia conforme se detalla a $\begin{array}{lllllllllllll}c & 0 & n & t & i & n & u & a & c & i & o & n & \text { : }\end{array}$

Jornadas de Formación e Innovación Docente del Profesorado | № 2 (2019) Esta obra se distribuye con la licencia Creative Commons Reconocimiento-NoComercial-SinObraDerivada Internacional (CC BY-NC-ND 4.0.) 
Derecho de Empresa

Concepto.

\author{
Empresa y Empresario \\ Sujetos y derechos.

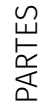

CLASES

- Mercantiles

- De capital

- Personalistas

$\underline{\text { Instrumentos tráfico Jurídico }}$

Títulos y valores

Derecho Concursal

Figura 2: Mapa de contenido inicial

Inicialmente se comprobó que el mapa primigenio realizado por los Alumnos adolecía del conocimiento del contenido del temario de la asignatura que se impartiría a lo largo del Curso, sin embargo ya advertía, para aquellos Alumnos de primera convocatoria, que a pesar de su desconocimiento en la materia, eran capaces de concebir y estructurar unas bases mínimas de clasificación de contenidos, y para aquellos Alumnos de convocatorias más avanzadas, denotaba que aun teniendo conocimiento de la materia, su clasificación mental de contenidos podía ser igual de aventurada que los primeros, lo que demostraba que ambos grupos, unos por desconocimiento, otros por error en la adquisición de la comprensión, partían del mismo mapa conceptual, lo cual resultaba una variable relevante para esta experimentación, por cuanto que, tantos los Alumnos ex novo, como los más veteranos parecían tener el mismo nivel de conocimiento, errado o no, pero en el mismo estadio.

Posteriormente, una vez impartida la materia, el mapa de contenido definitivo quedó desarrollado de la siguiente manera, a tenor de los resultados obtenidos en la evaluación del Ciclo de Mejora en el Aula, arrojando el mapa inicial las lagunas de conocimiento disciplinar de los Alumnos que había sido necesario complementar: 


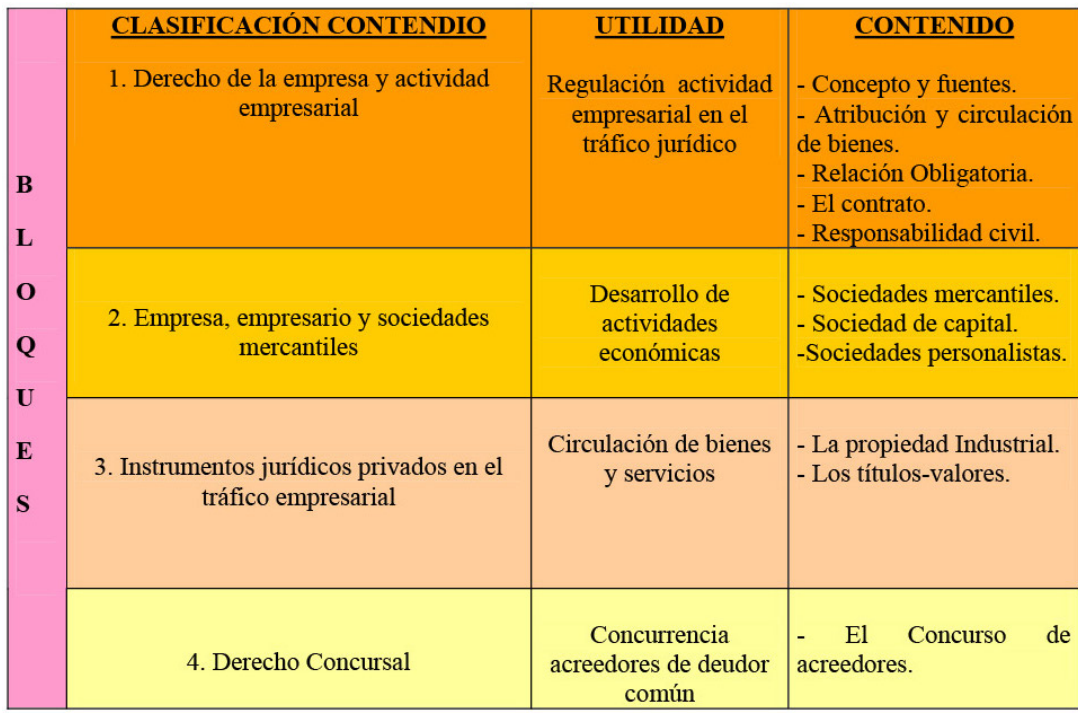

Figura 3: Mapa de contenido final.

\section{Modelo metodológico posible y secuencia de actividades programada, partiendo de los problemas seleccionados}

Como se ha indicado anteriormente, la novedad de este Ciclo de Mejora en el Aula ha consistido en sustituir el cuestionario inicial y final por la elaboración de mapas de contenidos de la materia por parte de los Alumnos que se apoyarán en la elaboración y resultado final de una actividad consistente en la creación de su propia empresa. A partir del análisis de los mapas iniciales se ha elaborado una escalera inicial con los diferentes modelos mentales de los estudiantes y sus porcentajes y se ha comparado con la escalera que se ha construido a partir del mapa final y el trabajo desarrollado en el capo práctico real.

Para ello se ha tenido en cuenta también los siguientes parámetros:

- Las conductas más significativas y en las emociones sentidas, tanto positivas como negativas. 
- El análisis de creencias que están asociadas a esas emociones.

- La aplicación el modelo metodológico diseñado a tal fin: $\mathrm{H}$ (Hipótesis) + P (Problema) + I (Investigación) + C (Conclusiones)

Conforme al método individual diseñado, se ha desarrollado en base a preguntas claves $\mathrm{H}$ (Hipótesis) que se han convertido intrínsicamente en interesantes para el alumno P (Problema) y que han despertado su curiosidad e inducción a la investigación I (Investigación) con la finalidad de resolver problemas en base a las conclusiones obtenidas en el campo práctico forense C (Conclusiones).

De esta manera se hacía necesario que los Alumnos adquirieran herramientas de aprendizaje que perdurasen a lo largo del tiempo y pudieran exportar a otras disciplinas o circunstancias distintas. Este ciclo de mejora ha propiciado esta vía, al incluir tanto las clases prácticas como teóricas, pues han estado estratégicamente inmersas las unas con las otras y reflejadas en las investigaciones y conclusiones del modelo metodológico, ya que se trata de reflexionar sobre la dimensión epistemológica del derecho mientras se resuelve un caso jurídico real pensando en las implicaciones que tiene en un caso concreto, en el que estoy implicado.

Para ello, los alumnos han ido creando -temporalmente con el desarrollo de las clases su propia empresa-, mediante fases de un modelo de investigación que ha sido el eje del trabajo principal, de manera que al ir abordando los problemas que se derivan de la creación de la empresa se han ido construyendo los contenidos conceptuales. Este ha sido el núcleo de trabajo para ir construyendo la teoría de la materia disciplinar. Por ello, se ha tratado de un método (M) de aprendizaje basado un modelo de investigación, aunque pudiera confundirse aparentemente con un aprendizaje basado en proyectos (ABP), dado que 
la investigación (I) y búsqueda de comprensión en la teoría ha sido el medio para resolver el problema $(P)$ ¿Qué empresa crear? Pregunta clave que sin duda suscitaba a los Alumnos la Hipótesis planteada $(\mathrm{H})$ ¿Para qué crear una empresa?:

H (Hipótesis $)+$ P (Problema $)+$ I (Investigación $)+C$ (Conclusiones)

Esta posible aparente confusión a la que se pudiera llegar entre este método de aprendizaje basado en un modelo de investigación con otro modelo de aprendizaje basado en proyectos (ABP), no es casual sino causal, pues se ha llevado a cabo conscientemente por el docente para determinar la variable que más había influido en la elección de los Alumnos, esto es, si conseguir crear una empresa como un propio proyecto, lo que hubiese supuesto un modelo de aprendizaje basado en proyectos (ABP), o si por el contrario la causa real del Alumno en investigar ¿para qué crear una empresa y cual? ha sido la causa lógica de la acción en el proceso de investigación para resolver el problema que le suscitaba tal hipótesis como preguntas claves que se han convertido intrínsicamente en interesantes para el Alumno hasta el punto de hacerle consciente de su capacidad para no solo resolver el problema sino que su propia solución le ha desvelado que es apto para hacer de una solución un proyecto. Y esta segunda variable ha sido a ciencia cierta la que ha predominado en esta experimentación.

Por ello el temario del contenido de la asignatura objeto del presente Ciclo de Mejora en el Aula ha constituido los problemas sobre los que se ha ido trabajando y que han estado asociados a la creación de la empresa. Se trata de orientar a los estudiantes en su propio proceso constructivo del conocimiento teórico para una aplicación práctico real, que ha argumentado la utilidad de la propia materia a comprender y justificado en primer oren de cosas el porqué conocerla y estudiarla, al margen de la 
calificación final que ha pasado en este caso a una meta secundaria y sin embargo consustancial con la primera.

La actividad a desarrollar, consistente en la creación por parte de los Alumnos de su propia empresa, se ha llevado a cabo intentado crear un entorno de aprendizaje natural que comparto con la idea recogida en el capítulo "¿Cómo dirigen la clase?" del manual Lo que hacen los mejores profesores universitarios. En primer lugar como componente esencial, al propio alumnado, sus características, su individualización, su grado de conocimiento y sus capacidades y habilidades. En segundo lugar, y consustancialmente unido al propio método de aprendizaje, debe también el docente hacer una autoevaluación de su grado de conocimiento en el campo práctico real, pues dificilmente se puede acercar al alumnado a un entorno de aprendizaje natural cuando el docente carece de ese complemento de conocimiento consistente en saber aplicar sus conocimientos teóricos a la vida real, y el resultado y efectos prácticos de los mismos.

\section{Cuestionario inicial-final para hacer un seguimiento de la evolución de los estudiantes en relación con los problemas clave}

Los Alumnos han ido constituyendo el proyecto empresarial por fases, libremente creadas y clasificadas por ellos, y consecuentes con el desarrollo del contenido de la materia impartido en clase de manera que han ido conformando el mapa de contenidos con la utilidad del mismo al caso real. En este sentido, y en coherencia con la clasificación de contenidos, han clasificado la creación del proceso empresarial en las siguientes etapas, que se han conformado por grupos de Alumnos bajo la libertad de su afinidad al perfil de las mismas, pero conscientes de que las distintas etapas guardan un orden de prelación que hacen depender una de la siguiente y así correlativamente, de 
manera que el trabajo de un grupo beneficiaría al siguiente, lo que les ha conducido a una cohesión y cooperación entre todos los Alumnos y la intercomunicación de contenidos, con un mismo objetivo, lo que ha cerrado sin duda esta investigación experimental de manera completa y de manera bidireccional consolidando el compromiso entre Alumnos y docente:

\section{CONCLUSIONES}

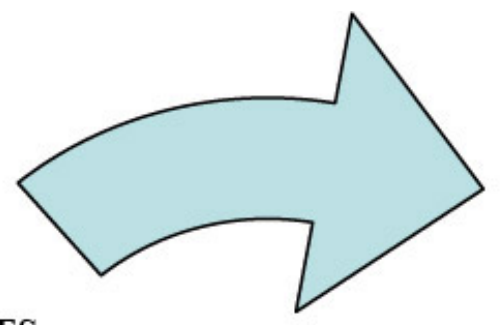

\section{HIPÓTESIS}

Y PROBLEMA

Valores

¿Para qué crear una

Valos empresa? ¿ y cual?

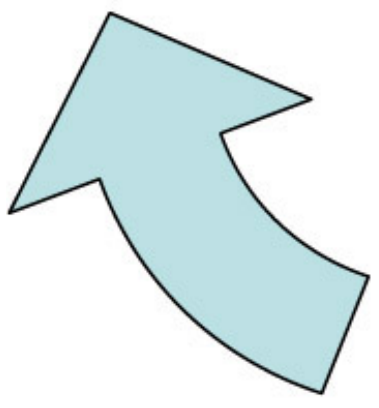

INVESTIGACIÓN

Ideas de negocio

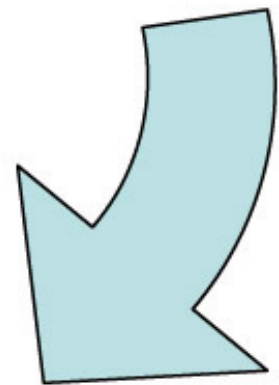

Objetivo, Misión, Visión

Figura 3. Actividad desarrollada en el diseño metodologicó.

Como recoge el libro Dar clase con la boca cerrada, en su capítulo "Experiencias que enseñan, crear experiencias para el aprendizaje", una secuencia preparada correctamente de preguntas puede reconducir el pensamiento hacia el razonamiento necesario para concluir la solución a un problema. 
Con esta premisa, se inició el Ciclo de Mejora en el Aula ¿Para que crear una empresa? y ¿cuál?

El resultado fue la constitución por parte de los Alumnos de la Mercantil iLawyer S.L.P, como una innovadora sociedad profesional de Abogados centrada en la asistencia de una plataforma virtual, determinando previamente los puntos esenciales del mercado para esta actividad empresarial evaluando de manera óptima cada criterio con la finalidad de la expansión de su prestación de servicios a nivel nacional como oportunidad de negocio, así como la financiación para su creación. Constituyeron mediante escritura pública la Sociedad y sus Órganos de Administración, sus Estatutos, procedieron a su inscripción y registro de derechos marcarios, realizaron el balance contable de un ejercicio anual completo, analizaron el resultado final y seleccionaron los tipos de contratos que regularían sus relaciones en el tráfico mercantil.

En ese proceso previo de determinación se valoraron por parte de los Alumnos, el objetivo, la misión, la visión de negocio y los valores.

\section{Aplicación del CMD}

\section{Resumen}

Conforme al sistema de la evaluación, para conocer y analizar los modelos mentales de los estudiantes hemos elaborado el cuestionario anteriormente referido para valorar los modelos de los alumnos, analizando según la escalera del grado de conocimiento antes y después de la experimentación las consecuencias didácticas de los cuestionarios que se examinan correlativamente con el siguiente resultado: cuál?

Primer cuestionario: ¿Para qué crear una empresa y Jornadas de Formación e Innovación Docente del Profesorado | № 2 (2019) Esta obra se distribuye con la licencia Creative Commons 
La actividad se realizó en 30 minutos antes de explicar la materia consistente en el Tema 1 del primer Bloque de contenidos clasificado, "Empresa, concepto y Fuentes". Después durante una hora y media se explicó el contenido del temario y se finalizó en la primera hora y media de clase del día siguiente, pero no se pasó nuevamente el cuestionario hasta finalizar el temario completo pues, como se ha indicado anteriormente, la novedad de este ciclo de mejora en el Aula ha consistido en sustituir el cuestionario inicial y final por la elaboración de mapas de contenidos de la materia por parte de los estudiantes. A partir del análisis de los mapas iniciales se ha construido una escalera inicial con los diferentes modelos mentales de los estudiantes y sus porcentajes, y se ha comparado con la escalera que se ha construido a partir del mapa final aplicado a la creación por parte de los Alumnos de su propia Empresa. Todo ello ha permitido un seguimiento durante el proceso al analizar los mapas intermedios y el resultado final aplicado a un caso práctico real.

\section{Evaluación del Aprendizaje}

Análisis comparativo de los cuestionarios inicial y final por sustitución del segundo ciclo de Mejora:

Cuestión inicial: ¿Para qué crear una empresa y cual?

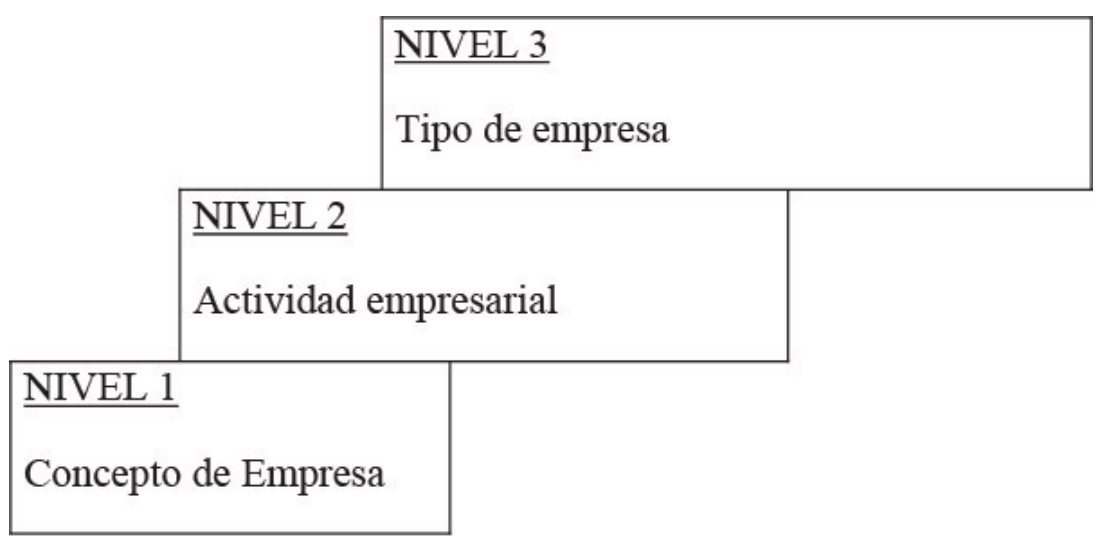

Figura 5: Escalera de aprendizaje.

Jornadas de Formación e Innovación Docente del Profesorado | № 2 (2019) Esta obra se distribuye con la licencia Creative Commons 
Nivel 1: Reconoce el concepto de empresa. $50 \%$ de los Alumnos. I Resultado posterior $100 \%$

Nivel 2: Identifica la actividad empresarial. $25 \%$ de los Alumnos. I Resultado posterior $80 \%$

Nivel 3: Clasifican tipos de empresas y su finalidad. $10 \%$ de los Alumnos. I Resultado posterior 70\%

En el desarrollo real de las actividades las consecuencias didácticas obtenidas se obtuvieron en un clima de trabajo compartido y participativo en igualdad de condiciones entre la actuación docente y el aprendizaje del Alumnado, con cierta desconfianza generalizada atendiendo a la peculiaridad de que la mayoría de los Alumnos que se encontraban en convocatorias avanzadas ya agotadas, por lo que existía inicialmente un ambiente reacio y desconfiado en la creencia de un posible cambio metodológico que propiciase un resultado favorable distinto al obtenido hasta ahora por ellos. Este ha sido un elemento fundamental en el desarrollo de la aplicación del ciclo de mejora en el Aula, por cuanto los Alumnos se sintieron participes del propio proyecto con un prejuicio o barrera mental inicial que se fue superando en las primeras semanas de clase eliminando la diferencia de estatus Profesor-Alumno. El conocimiento se fue creando de manera equitativa, entre los propios resultados de la actividad facilitados por los Alumnos y completado con el conocimiento teórico llevado al campo real del Profesor. Esto ha propiciado que los Alumnos vuelva a creer en sus capacidades, asumiéndolas, sabiéndose competentes para formar un conocimiento, con posibilidad de aportar al mismo sus propias ideas, y no menos importante creyendo en ellos mismos como medio para aprender y tener criterio propio con el objetivo de conseguir un resultado deseado en la superación de la asignatura en el que los Alumnos han encontrado el sentido del propio control de su dirección. 
En las clases sucesivas, desde esta perspectiva, los Alumnos han participado en el desarrollo de las clases con una libertad distinta al inicio del Ciclo de Mejora, sintiéndose más libres en su pensamiento, en sus expresiones y opiniones en cuanto a las explicaciones que se fueron desarrollando hasta completar el temario del Programa, y definitiva con la confianza suficiente que el conocimiento de la causa les daba para expresar sus dudas o conclusiones. El ambiente final del Curso se cerró con una posición de igualdad entre partes, donde el objetivo final era el mismo, la adquisición del conocimiento positivo, evaluación compartida en el examen final de la Asignatura en cuanto al conocimiento adquirido por los Alumnos y el conocimiento impartido por el Profesor. Una misma meta para ambos.

En cuanto a la valoración final del aprendizaje alcanzado en términos generales observo una evolución favorable de los Alumnos conforme a la comparación de ambas escaleras que se refleja objetivamente en los avances conseguidos, puesto que han superado la asignatura el $99 \%$ de los Alumnos que han participado en el presente $\mathrm{Ci}$ clo de Mejora en el Aula. Los Alumnos han sido conscientes de manera escenificada de que su zona de desarrollo próximo alcanza un diámetro mayor que al principio del cuatrimestre porque en definitiva han encontrado la utilidad del conocimiento, lo que ha justificado su participación, esfuerzo y voluntariedad con un resultado positivo y palpable acorde al deseado. 


\section{Evaluación del CMD puesto en práctica}

\section{Cuestiones a mantener y cambios a introducir para el futuro}

Los resultados han sido objetivamente tangibles, fruto de la libertad y voluntariedad de los Alumnos en participar y creer en el proyecto experimental del Ciclo de Mejora en el Aula participado activamente.

En cuanto a los aspectos que se van a mantener como cambios habituales en las clases: la consciencia consecuente de los Alumnos del método y su justificación así como de su implicación en el mismo de cara a un objetivo favorable de su utilidad, manteniendo el planteamiento previo de P (Problema) + H (Hipótesis) + I (Investigación) + C (Conclusiones), al inicio de cada programa y que sean los Alumnos los que activamente vayan realizándolo conforme a una aplicación práctico-real. Precisamente este fue uno de los cambios a introducir en futuros ciclos de mejora que se contempló por parte de esta autora en su anterior Artículo, y sin duda su introducción ha sido positiva en el resultado final

En referencia a los cambios a introducir para un futuro Ciclo de Mejora, en el caso de la actividad propia de la constitución de la empresa por los propios Alumnos, es pertinente realizar una evaluación continua que permita ir valorando la misma por fases y no en su etapa final, a fin de ir corrigiendo errores en su elaboración que permita una mejor consolidación en la prelación de contenidos de cara a las fases correlativas de la actividad. 


\section{Aspectos de la Experiencia que se pretenden aplicar a toda la práctica docente habitual}

Transmitir y aplicar herramientas de aprendizaje trasladables a cualquier disciplina. La adquisición de conocimiento razonado para aplicarlo en el campo práctico concreto, permitiendo un estudio duradero en el tiempo siendo un conocimiento estructurado y aplicado no memorizado, sino conscientemente útil en cuanto a su aplicación práctico real en la vida cotidiana, generando así la necesidad de adquirir el propio conocimiento como una solución futura a distintos problemas que se les pueda platear, haciendo a los Alumnos más libres en relación a sus decisiones teniendo criterio sostenible para ello.

\section{Principios didácticos argumentados que han guiado la experiencia y que deben permanecer en el futuro}

En cuanto al aprendizaje de los/as alumnos/as: Como elemento esencial la necesaria individualización de los alumnos y el análisis como premisa de sus esquemas mentales, que en este Grupo en particular ha sido dificil de desestructurar por la desconfianza natural adquirida de gran parte de los Alumnos que se encontraban en convocatorias avanzadas ya agotadas y tenían una actitud reacia hacia la asignatura objeto del proyecto experimental. Para ello es fundamental partir del grado de conocimiento inicial de los estudiantes para acercar la disciplina a ellos y construir desde ese estadio el conocimiento de la materia.

Por ello, es necesario analizar el contexto y circunstancias personales en el que cada Alumno va a adquirir el conocimiento puesto que las mismas incidirán de manera más o menos positiva y directa en la adquisición del conocimiento. 
En cuanto a los contenidos de enseñanza: la creación de mapas de contenidos clasificados y aplicados temporalmente a un caso práctico de manifiesta utilidad coherente con las preguntas e inquietudes que la materia suscita en los Alumnos para justificar su necesidad.

\section{En cuanto a la metodología y actividades:}

El desarrollo de preguntas claves que activen el modelo metodológico diseñado dirigido a actividades experimentales en el Aula que hagan real lo teórico y devenguen la necesidad de la investigación propicia conducente a la adquisición de conocimientos que justifiquen y argumenten la propia utilidad de la disciplina en cuestión.

La interacción de contenidos y de las herramientas de aprendizaje adquiridas trasladables a cualquier orden y materia como instrumento de conocimiento y utilidad multidisciplinar.

En cuanto a la evaluación: para su mejora y desarrollo en el resultado final de la actividad, quizá una evaluación conjunta en tiempo real posibilite una mejor consolidación de contenidos de cara a la perfección de su adquisición con una evaluación final más favorable.

Jornadas de Formación e Innovación Docente del Profesorado | № 2 (2019) Esta obra se distribuye con la licencia Creative Commons Reconocimiento-NoComercial-SinObraDerivada 


\section{Referencias Bibliográficas.}

Bain, K. (2004) Lo que hacen los mejores profesores universitarios. Valencia: Publicaciones Universidad de Valencia.

Finkel, D. (2008). Dar clases con la boca cerrada. Valencia: Publicaciones Universidad de Valencia.

Porlán, R. (2017). Enseñanza Universitaria, Cómo mejorarla. Madrid: Editorial Morata.

Jornadas de Formación e Innovación Docente del Profesorado | № 2 (2019) Esta obra se distribuye con la licencia Creative Commons 